\title{
QuickNGS elevates Next-Generation Sequencing data analysis to a new level of automation
}

Prerana Wagle 1 , Miloš Nikolić ${ }^{1,2}$ and Peter Frommolt ${ }^{1 *}$

\begin{abstract}
Background: Next-Generation Sequencing (NGS) has emerged as a widely used tool in molecular biology. While time and cost for the sequencing itself are decreasing, the analysis of the massive amounts of data remains challenging. Since multiple algorithmic approaches for the basic data analysis have been developed, there is now an increasing need to efficiently use these tools to obtain results in reasonable time.

Results: We have developed QuickNGS, a new workflow system for laboratories with the need to analyze data from multiple NGS projects at a time. QuickNGS takes advantage of parallel computing resources, a comprehensive backend database, and a careful selection of previously published algorithmic approaches to build fully automated data analysis workflows. We demonstrate the efficiency of our new software by a comprehensive analysis of 10 RNA-Seq samples which we can finish in only a few minutes of hands-on time. The approach we have taken is suitable to process even much larger numbers of samples and multiple projects at a time.
\end{abstract}

Conclusion: Our approach considerably reduces the barriers that still limit the usability of the powerful NGS technology and finally decreases the time to be spent before proceeding to further downstream analysis and interpretation of the data.

Keywords: Next-Generation Sequencing, Batch processing, Data management, High-performance computing, Analysis workflow

\section{Background}

Next-Generation Sequencing (NGS) has become the method of choice for molecular genetic analyses such as transcriptome profiling (RNA-Seq, miRNA-Seq), chromatin immunoprecipitation followed by sequencing (ChIP-Seq) as well as resequencing of complete genomes. Numerous software packages designed to analyze massive amounts of NGS data are now publicly available. Preprocessing of NGS data typically takes advantage of a complex hardware architecture composed of, for instance, a parallel compute cluster, a database server and a web server. As this requires specialized IT skills, the widespread access to NGS technology is still hampered by technical barriers. The primary data analysis is therefore often centralized into core

\footnotetext{
* Correspondence: peter.frommolt@uni-koeln.de

'Bioinformatics Core Facility, CECAD Research Center, University of Cologne, Joseph-Stelzmann-Str. 26, 50931 Cologne, Germany

Full list of author information is available at the end of the article
}

laboratories which face the challenge of using a reasonable selection out of the available software packages to process a growing flow of newly produced data.

We introduce a new framework named QuickNGS which can be operated by IT staff in bioinformatics core labs to process vast amounts of data provided by their end users, typically experimental scientists. QuickNGS enables a major decrease in time and effort put into the primary analysis of NGS data, thus contributing to the further evolution of NGS into a standard tool with high accessibility to researchers.

\section{Implementation}

QuickNGS enables rapid and professional data analysis for the aforementioned major applications of NGS in a batch-like operation mode. The core of the QuickNGS workflow is formed by a comprehensive MySQL database used to organize sample metadata such as species, library type and batch information as well as the analysis 
a

QuickNGS - Sample upl... $\times$ +

* athen.cecad.uni-koeln.de/quickngs/panfs/db/samples.php

- C] 8 - coogle

Q日自自侖三

Quick NGS All-in-one data processing for Next-Generation Sequencing

QuickNGS database

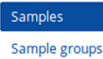

Species

Laboratories

Institutions

Status

b

Laboratory: Peter Frommolt

而 773 w 2
Instructions

Click + to add new samples, 通 to delete or to edit a sample from the database.

Scroll to the right to see the complete file paths of the samples.

\begin{tabular}{|c|c|c|c|c|c|}
\hline \pm & ID & Label & Application & Lib & Species \\
\hline 面: & 809 & 9 580_forward_test_milos & RNA-Seq & $S R$ & mus_musculus \\
\hline 而, & 781 & 1 atf3a_2 & RNA-Seq & $S R$ & drosophila_melanogaster \\
\hline 而 & 780 & 0 atf3a_1 & RNA-Seq & $S R$ & drosophila_melanogaster \\
\hline 而 & 779 & 9 rel_2 & RNA-Seq & $S R$ & drosophila_melanogaster \\
\hline 而 & 778 & 8 rel_1 & RNA-Seq & $\mathrm{SR}$ & drosophila_melanogaster \\
\hline 而 & 777 & 7 foxo_2 & RNA-Seq & $S R$ & drosophila_melanogaster \\
\hline 而, & 776 & 6 foxo_1 & RNA-Seq & SR & drosophila_melanogaster \\
\hline 而; & 775 & 5 atf376_2 & RNA-Seq & $S R$ & drosophila_melanogaster \\
\hline 而 & 774 & 4 atf376_1 & RNA-Seq & $S R$ & drosophila_melanogaster \\
\hline
\end{tabular}

RNA-Sea

R drosovhilla melanogaster

Q(1) QuickNGS - Run status - Mozilla Fire fox

QuickNGS-Run status $\quad x$

$\leftarrow$ athen.cecad.uni-koeln.de/quickngs/panfs/db/status.php

- c 8 - Google

Q日自会三 Quick NGS All-in-one data processing for

Status of recent NGS pipeline runs

\begin{tabular}{|c|c|c|c|c|c|c|c|c|c|}
\hline RunID & Username & Application & Species & \# Samples & PI & Module & Started & Finished & Status \\
\hline 1507 & pwagle & RNA-Seq & mus_musculus & 16 & Bjoern Schumacher & PreProcessing & 2014-10-01 15:51:07 & 2014-10-01 15:51:43 & Finished \\
\hline 1507 & pwagle & RNA-Seq & mus_musculus & 16 & Bjoern Schumacher & TophatCufflinks & 2014-10-01 15:51:43 & & Running \\
\hline 1506 & pwagle & RNA-Seq & caenorhabditis_elegans & 24 & Bjoern Schumacher & PreProcessing & 2014-10-01 13:51:15 & 2014-10-01 13:52:16 & Finished \\
\hline 1506 & pwagle & RNA-Seq & caenorhabditis_elegans & 24 & Bjoern Schumacher & TophatCufflinks & 2014-10-01 13:52:16 & & Running \\
\hline 1505 & pwagle & RNA-Seq & mus_musculus & 16 & Bjoern Schumacher & PreProcessing & 2014-10-01 12:51:07 & $2014-10-0112: 51: 44$ & Finished \\
\hline 1505 & pwagle & RNA-Seq & mus_musculus & 16 & Bjoern Schumacher & TophatCufflinks & 2014-10-01 12:51:44 & & Running \\
\hline 1504 & frommolp & RNA-Seq & drosophila_melanogaster & 10 & Peter Frommolt & PreProcessing & 2014-10-01 11:51:04 & $2014-10-0111: 51: 24$ & Finished \\
\hline 1504 & frommolp & RNA-Seq & drosophla_melanogaster & 10 & Peter Frommolt & TophatCufflinks & 2014-10-01 11:51:24 & 2014-10-01 14:23:15 & Finished \\
\hline 1504 & frommolp & RNA-Seq & drosophila_melanogaster & 10 & Peter Frommolt & Wiggles & 2014-10-01 14:23:15 & 2014-10-01 15:09:22 & Finished \\
\hline 1504 & frommolp & RNA-Seq & drosophlla_melanogaster & 10 & Peter Frommolt & ReadCount & 2014-10-01 14:23:17 & & Running \\
\hline 1503 & pwagle & RNA-Seq & drosophlia_melanogaster & 16 & Mirka Uhlirova & PreProcessing & 2014-09-30 14:51:07 & 2014-09-30 14:51:45 & Finished \\
\hline 1503 & pwagle & RNA-Seq & drosophila_melanogaster & 16 & Mirka Uhlirova & TophatCufflinks & 2014-09-30 14:51:45 & $2014-10-0115: 24: 02$ & Finished \\
\hline 1503 & pwagle & RNA-Seq & drosophlla_melanogaster & 16 & Mirka Uhlirova & Wiggles & 2014-10-01 15:24:02 & & Running \\
\hline 1503 & pwagle & RNA-Seq & drosophila_melanogaster & 16 & Mirka Uhlirova & ReadCount & $2014-10-0115: 24: 02$ & & Running \\
\hline 1500 & frommolp & RNA-Seq & homo_sapiens & 7 & Matthias Fischer & PreProcessing & 2014-09-30 10:51:05 & 2014-09-30 10:51:24 & Finished \\
\hline 1500 & frommolp & RNA-Seq & homo_sapiens & 7 & Matthias Fischer & TophatCufflinks & 2014-09-30 10:51:25 & 2014-09-30 13:39:55 & Finished \\
\hline 1500 & frommolp & RNA-Seq & no_sapiens & 7 & atthias Fischer & Wiggles & 2014-09-30 13:39:55 & 2014-09-30 15:58:12 & Finished \\
\hline 1500 & frommolp & RNA-Seq & homo_sapiens & 7 & Matthlas Fischer & ReadCount & 2014-09-30 13:39:55 & 2014-09-30 15:59:33 & Finished \\
\hline 1500 & frommolp & RNA-Seq & homo_sapiens & 7 & Matthias Fischer & QC & $2014-09-3015: 59: 33$ & $2014-09-3017: 17: 44$ & Finished \\
\hline 1500 & frommolp & RNA-Seq & homo_sapiens & 7 & Matthias Fischer & PostProcessing & $2014 \cdot 09-3017: 17: 44$ & $2014-09-3017: 28: 56$ & Finished \\
\hline 1499 & pwagle & RNA-Seq & caenorhabditis_elegans & 9 & Aleksandra Trifunovic & PreProcessing & $2014-09-30$ 10:01:05 & 2014-10-01 08:38:01 & Finished \\
\hline 1499 & pwagle & RNA-Seq & caenorhabditis_elegans & 9 & Aleksandra Trifunovic & TophatCufflinks & 2014-09-30 10:01:32 & 2014-09-30 15:28:00 & Finished \\
\hline 1499 & pwagle & RNA-Seq & norhabditis_elegans & 9 & Aleksandra Trifunovic & Wiggles & 2014-10-01 08:38:01 & 2014-10-01 09:01:45 & Finished \\
\hline 1499 & pwagle & RNA-Seq & caenorhabditis_elegans & 9 & Aleksandra Trifunovic & ReadCount & 2014-10-01 08:38:01 & $2014-10-0111: 46: 50$ & Finished \\
\hline 1499 & pwagle & RNA-Seq & & 9 & & & $2014-10-0111: 46: 51$ & $2014-10-01 \quad 14: 12: 40$ & Finist \\
\hline 1499 & pwagle & RNA-Seq & caenorhabditis elegans & 9 & Aleksandra Trifunovic & DiffExpression & 2014-10-01 14:12:41 & 2014-10-01 14:14:02 & Finished \\
\hline
\end{tabular}

Fig. 1 (See legend on next page.) 
(See figure on previous page.)

Fig. 1 a The QuickNGS database contains meta information on samples (species, application, file locations, sample labels, lab name, library type, batch information) and sample groups (samples which are forming groups to be compared). Both can be efficiently organized by an intuitive web interface. New samples and sample groups can be inserted (Additional file 4) by following the "+ "button. $\mathbf{b}$ The status page on the QuickNGS database monitors time, user information and current status of each QuickNGS module on a clearly arranged website, enabling password-protected interrogation of the current status from any working location, including mobile access

results. The system operators can use the QuickNGS database interface (Fig. 1a) to upload metadata and monitor the status of QuickNGS analysis runs from any location (Fig.1b). On the other hand, the purpose of the QuickNGS web interface is to deliver web reports on the analysis results to the end users who can use their personal login and password to browse the results plus a lot of useful on-top information (Fig. 2). The core results are provided as spreadsheet files which the user can download to a local computer.

As the sample metadata in the QuickNGS database are used to completely control the overall workflow, these have to be provided to the QuickNGS database before any analysis can be started. To achieve this, the file locations of the raw data first need to be saved into a text file (Additional file 4a). This file is then uploaded into the QuickNGS web interface (Additional file 4b) together with information on the library type, NGS application type, species of origin and the laboratory in which the input material has been generated. For each sample listed in the text file, the user is then asked to provide a human-readable sample label as well as batch information for the case that samples have been processed with different library preparation protocols or in different NGS runs (Additional file 4c). Finally, samples can be assigned to pairs and groups for comparative analysis, e.g. differential gene expression (Additional file $4 d)$. Subsequently, the raw data received from the sequencing center are linked into the QuickNGS stack directory which is then processed fully automatically. Thus, the only user action needed is providing sample information and linking the files to the stack directory which

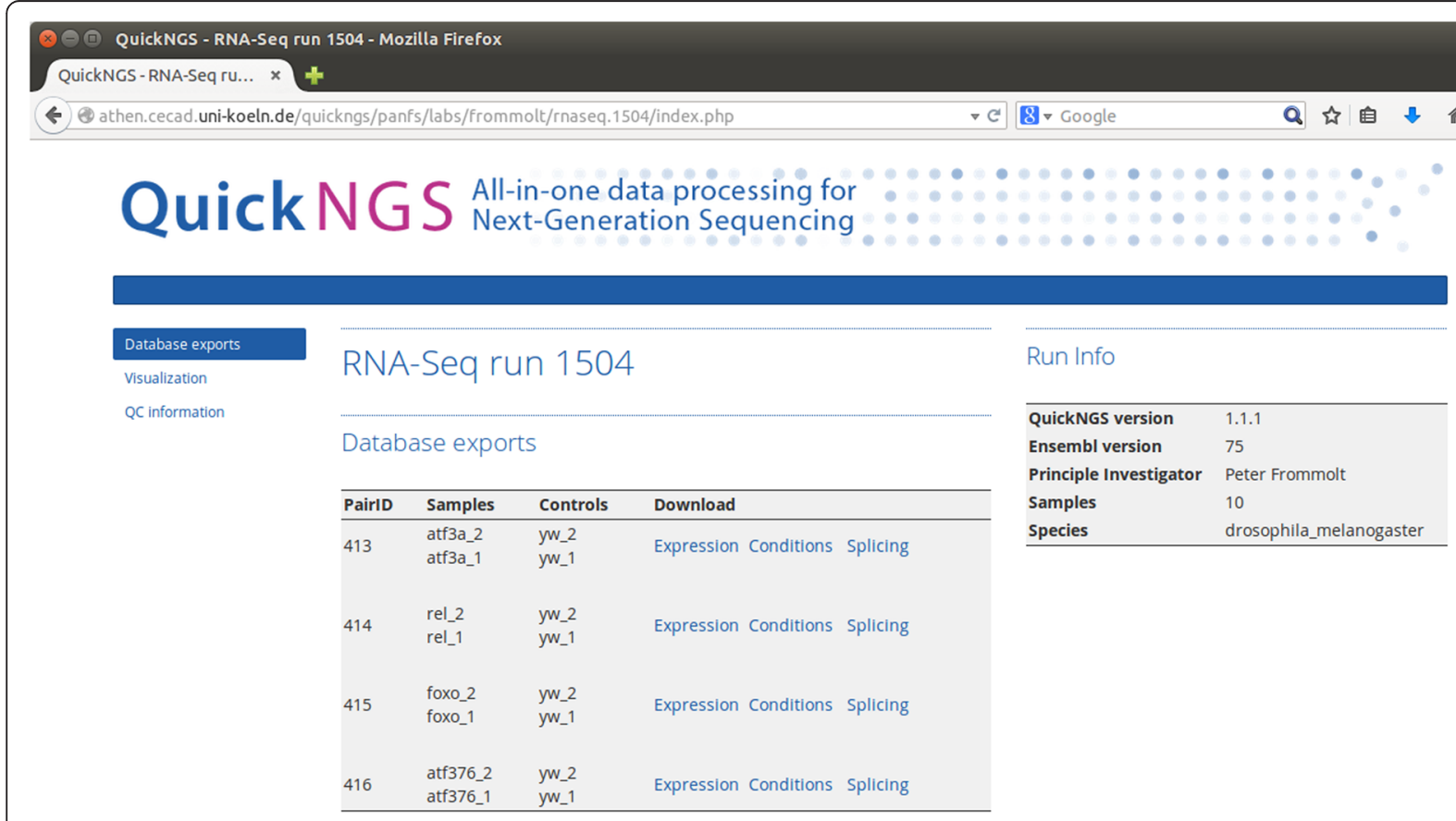

Fig. 2 Results report for the test run on 10 Drosophila RNA-Seq samples. After login, the interface provides links to the main database export files, QC statistics as well as visualisation plots and the user's personal password-protected UCSC genome browser track hub 
Table 1 Algorithms and software tools used by QuickNGS, version 1.1.0. The selection may be modified, updated or extended in future releases of QuickNGS, however, an up-to-date version of this table will be kept available online at the QuickNGS website

\begin{tabular}{|c|c|c|c|}
\hline & Tool & Version & Reference \\
\hline \multirow[t]{6}{*}{ RNA-Seq } & FastQC & 0.10 .1 & \\
\hline & Tophat2 & 2.0 .10 & [12] \\
\hline & Cufflinks2 & 2.1 .1 & [19] \\
\hline & DESeq2 & 1.4 .5 & [1] \\
\hline & DEXSeq & 1.10 .5 & [2] \\
\hline & UCSC Genome Browser & & [11] \\
\hline \multirow[t]{4}{*}{ miRNA-Seq } & FastQC & 0.10 .1 & \\
\hline & miRDeep2 & 0.0 .5 & [6] \\
\hline & DESeq2 & 1.4 .5 & [1] \\
\hline & UCSC Genome Browser & & [11] \\
\hline \multirow[t]{5}{*}{ ChIP-Seq } & FastQC & 0.10 .1 & \\
\hline & BWA & 0.7 .7 & [13] \\
\hline & MACS2 & 2.0.10 & [5] \\
\hline & MEME-ChIP & 4.10 .0 & [15] \\
\hline & UCSC Genome Browser & & [11] \\
\hline \multirow[t]{6}{*}{ WGS } & FastQC & 0.10 .1 & \\
\hline & BWA & 0.7 .7 & [13] \\
\hline & Samtools & 0.1 .19 & [13] \\
\hline & Delly & 2.0 .1 & [16] \\
\hline & SnpEff & 3.4 & [3] \\
\hline & UCSC Genome Browser & & [11] \\
\hline
\end{tabular}

both are trivial amounts of effort. The results produced by the workflow are saved back into the QuickNGS database and made accessible through a report on the web interface. This report comprises standard QC metrics (read counts, read quality, contamination, library quality,
QC plots, cluster analyses etc.) and results on typical datarelated research questions, for instance which genes are differentially expressed or differentially spliced, which genomic variations are unique to a sample compared to a control, which transcription factor binding motifs are enriched in a ChIP-Seq data set etc.. The results and report are generated fully automatically without any additional user action.

The analysis relies on widely adopted NGS analysis packages which are listed in Table 1 . For the core analysis of the raw data, we have carefully selected the most appropriate previously published software programs. The selection criteria were (1) performance in published and in-house benchmarking studies, (2) comprehensiveness of the analysis output, (3) quality of the implementation and steadiness of maintenance, and (4) popularity in the community. Our choice of bioinformatics software follows these criteria as far as possible. The code for QC and visualisation as well as for data management and the workflow itself is unique to QuickNGS. As a reference to genomic sequence and annotation, the system uses the miRBase [8] for the miRNA-Seq workflow and the Ensembl database [9] for all other applications. For instance, RNA-Seq or ChIP-Seq analyses can thus be carried out on data from any arbitrary organism listed on either Ensembl (69 species as of release 76) or EnsemblGenomes (54 metazoa, 38 plants, 52 fungi, 32 protists, 15270 bacteria as of release 23). The reference files are downloaded to the local system and updated automatically. The same applies to genomic annotation data which are retrieved using BioMart [4].

\section{Results and discussion}

\section{Test run on previously published RNA-Seq data}

To illustrate the practical use of our software, we have re-analyzed 10 RNA-Seq samples from a study on

Table 2 Reads statistics on the test data from [18] - read counts are given in multiples of $10^{6}(1 \mathrm{M}=1$ million reads). Duplicate removal was not performed because this was a single-read analysis. Two samples (yw_1 and atf376_1) were treated with ribo-zero, whereas for the remaining samples, there is a significant degree of contamination with ribosomal RNA. For all samples, about the half of the reads map to the original strand because all data origin from unstranded libraries

\begin{tabular}{llllllll}
\hline Label & \# Reads & \# Aligned & MapQ $\geq 30$ & Stranded & miRNA & rRNA & Other ncRNA \\
\hline yw_1 & $25.9 \mathrm{M}$ & $17.4 \mathrm{M}$ & $16.2 \mathrm{M}$ & $50.2 \%$ & $0.0 \mathrm{M}$ & $0.3 \mathrm{M}$ & $0.1 \mathrm{M}$ \\
yw_2 & $37.4 \mathrm{M}$ & $34.2 \mathrm{M}$ & $32.5 \mathrm{M}$ & $50.5 \%$ & $0.0 \mathrm{M}$ & $5.3 \mathrm{M}$ & $0.2 \mathrm{M}$ \\
atf376_1 & $26.9 \mathrm{M}$ & $20.8 \mathrm{M}$ & $19.3 \mathrm{M}$ & $50.3 \%$ & $0.0 \mathrm{M}$ & $0.1 \mathrm{M}$ & $0.1 \mathrm{M}$ \\
atf376_2 & $36.3 \mathrm{M}$ & $32.5 \mathrm{M}$ & $31.3 \mathrm{M}$ & $50.9 \%$ & $0.0 \mathrm{M}$ & $3.4 \mathrm{M}$ & $0.2 \mathrm{M}$ \\
foxo_1 & $37.6 \mathrm{M}$ & $34.1 \mathrm{M}$ & $32.6 \mathrm{M}$ & $50.0 \%$ & $0.0 \mathrm{M}$ & $2.9 \mathrm{M}$ & $0.3 \mathrm{M}$ \\
foxo_2 & $39.3 \mathrm{M}$ & $33.0 \mathrm{M}$ & $31.7 \mathrm{M}$ & $50.2 \%$ & $0.0 \mathrm{M}$ & $3.0 \mathrm{M}$ & $0.3 \mathrm{M}$ \\
rel_1 & $37.8 \mathrm{M}$ & $34.6 \mathrm{M}$ & $32.9 \mathrm{M}$ & $50.0 \%$ & $0.0 \mathrm{M}$ & $2.9 \mathrm{M}$ & $0.3 \mathrm{M}$ \\
rel_2 & $38.1 \mathrm{M}$ & $34.6 \mathrm{M}$ & $33.0 \mathrm{M}$ & $50.1 \%$ & $0.0 \mathrm{M}$ & $3.0 \mathrm{M}$ & $0.3 \mathrm{M}$ \\
atf3a_1 & $38.4 \mathrm{M}$ & $34.7 \mathrm{M}$ & $33.2 \mathrm{M}$ & $50.3 \%$ & $0.0 \mathrm{M}$ & $3.8 \mathrm{M}$ & $0.3 \mathrm{M}$ \\
atf3a_2 & $38.5 \mathrm{M}$ & $35.2 \mathrm{M}$ & $33.7 \mathrm{M}$ & $50.3 \%$ & $0.0 \mathrm{M}$ & $3.9 \mathrm{M}$ & $0.3 \mathrm{M}$ \\
\hline
\end{tabular}


transcription factors in Drosophila where 4 mutant conditions were compared to a control with two biological replicates [18]. After feeding the sample metadata into the QuickNGS database, we have linked the 20 FastQ files into the QuickNGS stack directory. These preparing steps took us an overall time of $2 \mathrm{~min}$. While waiting for the subsequent pipeline run to finish, we were able to monitor the current status of the respective modules using the status page on the QuickNGS database interface (Fig. 1b). The RNA-Seq workflow comprises an initial quality check using FastQC plus some software which is unique to QuickNGS. The basic data processing consists of a splicing-aware alignment using Tophat2 [12] followed by reference-guided transcriptome reassembly with Cufflinks2 [19]. Differential gene expression and differential exon usage are analyzed with DESeq2 ([1], Genome Biol) and DEXSeq ([2], Genome Res). After the processing finished overnight, we

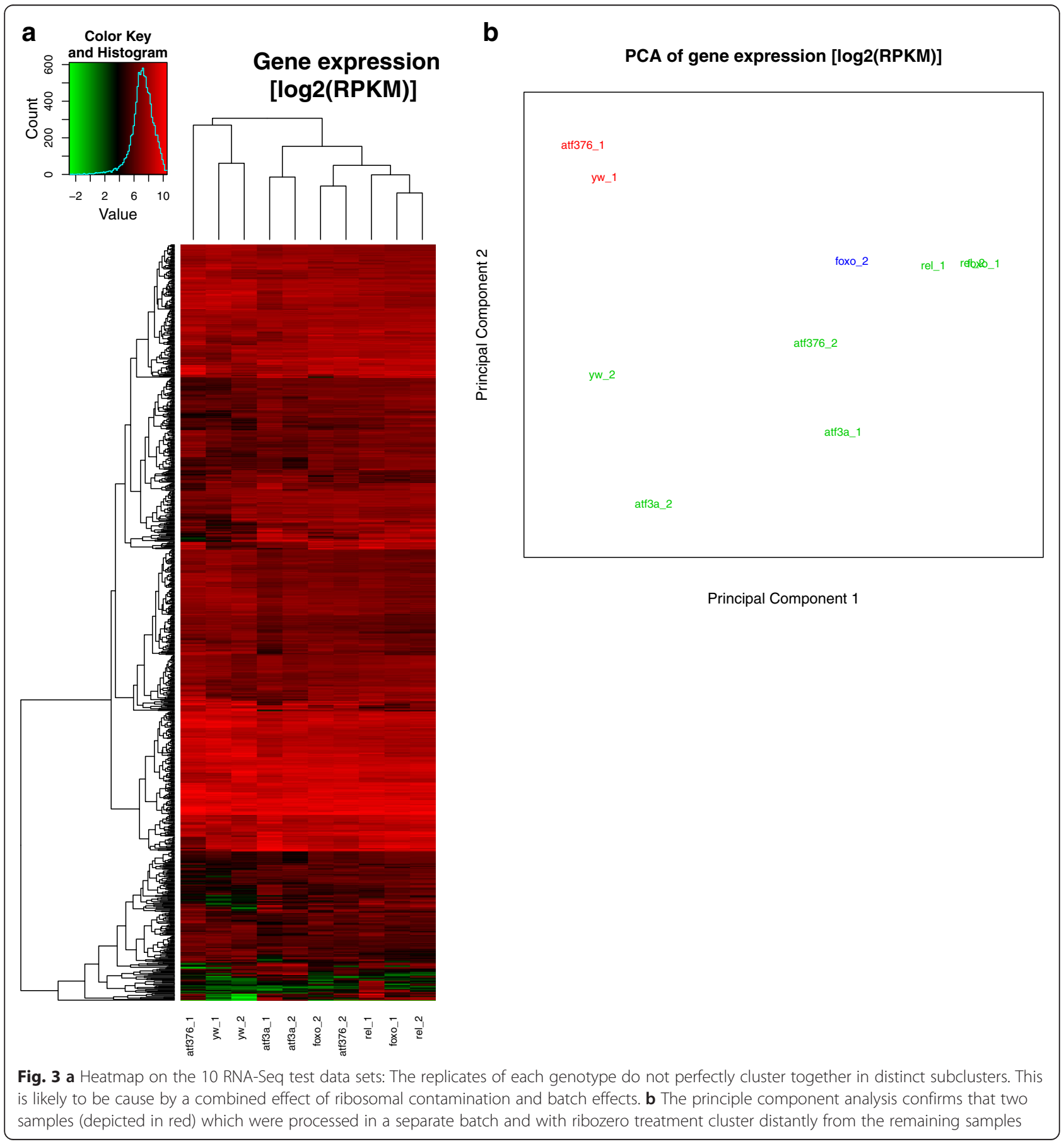


logged in to the QuickNGS user interface and found a report which summarizes all results of the QuickNGS workflow (Fig. 2). From the initial quality check, we received some basic read statistics (Table 2) as well as standard QC plots, a heat map (Fig. 3a) and a plot from a principle component analysis (Fig. 3b) for the 10 samples. The results of the core analysis for the comparison of atf3 mutants (atf3a_1 and atf3a_2) against controls (yw_1 and yw_2) are provided as Additional files. At thresholds 5 and 0.01 for fold-change and p-value, we get a set of 93 differentially expressed genes (Additional file 1) and a set of 168 differentially used exons (Additional file 2). Additional file 3 reports the p-values and fold-changes for differential gene expression (atf3a_1 and atf3a_2 compared to $y w \_1$ and $\left.y w \_2\right)$ together with those for the comparisons of the remaining three mutant conditions to control (atf376_1 and atf376_2, foxo_1 and foxo_2, rel_1 and rel_2, each compared against $y w \_1$ and $\left.y w \_2\right)$. On the web interface, the same three spreadsheet files are given also for these comparisons. All tables contain a comprehensive selection of genomic and functional annotation. Visualisation of the RNA-Seq wiggle files on the UCSC Genome Browser can be accessed by a hyperlink which uses a local password-protected track hub for the browser. The FastQ files for these test data are available from the NCBI Short Read Archive (SRA) at accession number SRP011390.

\section{Description of other QuickNGS workflows}

Although the current QuickNGS release also comprises workflows for miRNA sequencing, ChIP-Seq and wholegenome resequencing, we gave above a detailed description only for the RNA-Seq workflow. However, the same level of efficiency and automation is also achieved in all other QuickNGS workflows. The miRNA-Seq workflow comprises quantification and differential profiling of $3 p$ and $5 p$ mature miRNAs using miRDeep [6] as well as statistics on miRNA families. Differential miRNA expression is profiled with the DESeq2 package [1]. The
ChIP-Seq workflow takes advantage of BWA [14] for genomic alignment of the reads and uses MACS2 [5] for peak calling. Furthermore, QuickNGS identifies all genes which are $2000 \mathrm{bp}$ up- or downstream from the MACS2 peaks. The peak sequences are analyzed for enrichment of transcription factor binding motifs using MEMEChIP [15]. The results comprise lists of significant peaks and reports for motif enrichment. For the whole-genome resequencing workflow, finally, the software uses BWA for genomic alignment and calls single nucleotide polymorphisms with SAMtools [13] and structural variations with Delly [16]. The results are annotated and functionally classified with SNPeff [3]. Basic QC statistics and passwordprotected track hubs for the UCSC Genome Browser with direct hyperlinks for visualisation are part of all workflows. The QuickNGS database comes with ready-made metadata for additional test data which are available from the SRA at NCBI at accession numbers SRP043191 (miRNASeq), SRP007261 (ChIP-Seq) and SRP020555 (wholegenome resequencing). Additional modules dedicated to cancer genomics and more recent NGS applications such as CLIP-Seq (cross-linking immunoprecipitation followed by sequencing) are currently under development.

\section{Features of QuickNGS compared to other NGS workflow systems}

In order to elaborate how QuickNGS performs in comparison to other NGS workflow systems, we discuss the features that are unique to our solution as well as its limitations. The degree of automation in QuickNGS is much higher than, for instance, that of an appropriate workflow in popular data analysis frameworks like Galaxy [7], GenePattern [17] or Chipster [10]. This makes the system more efficient for the typical standard analyses, but also less flexible to modifications. In particular, our system enables an extreme reduction of the handson (not computation) time that staff have to spend for the basic NGS data analyses. Data processing for tens or

Table 3 Comparison of the technical features of QuickNGS to those of other NGS analysis workflow systems

\begin{tabular}{|c|c|c|c|c|}
\hline & QuickNGS & Galaxy & GenePattern & Chipster \\
\hline Setup & $\begin{array}{l}\text { Compute cluster plus DB and } \\
\text { web server }\end{array}$ & Client/server system & Client/server system & Client/server system \\
\hline Applications & $\begin{array}{l}\text { RNA-Seq, miRNA-Seq, ChIP-Seq, } \\
\text { Whole-Genome }\end{array}$ & Universal framework & RNA-Seq & $\begin{array}{l}\text { RNA-Seq, miRNA-Seq, } \\
\text { ChIP-Seq, Whole-Genome }\end{array}$ \\
\hline Database & Metadata and results & None & None & None \\
\hline Workflow automation & Full & Started in web interface & Started in web interface & Started in client software \\
\hline Reproducibility/Documentation & $\begin{array}{l}\text { Results kept in DB Version } \\
\text { tracking Logfiles }\end{array}$ & Workflow files & Workflow files & Workflow files \\
\hline Workflow flexibility & Requires shell programming & $\begin{array}{l}\text { Can be changed in } \\
\text { web interface }\end{array}$ & $\begin{array}{l}\text { Can be changed in web } \\
\text { interface }\end{array}$ & $\begin{array}{l}\text { Can be edited in client } \\
\text { software }\end{array}$ \\
\hline Purpose of user interface & $\begin{array}{l}\text { End-user access to the } \\
\text { analysis results }\end{array}$ & $\begin{array}{l}\text { Data import and start } \\
\text { of workflows }\end{array}$ & $\begin{array}{l}\text { Data import and start } \\
\text { of workflows }\end{array}$ & $\begin{array}{l}\text { Data import and start of } \\
\text { workflows }\end{array}$ \\
\hline
\end{tabular}


hundreds of samples can be initiated in less than $10 \mathrm{~min}$. While the subsequent analyses completely run in the background, they can be monitored on the status website and, once finished, the results are ready for immediate access by any scientist without specific IT skills. In contrast to all other systems, the QuickNGS database is capable of organizing sample meta information along with the analysis results, enabling a high degree of reproducibility and documentation of what analyses have been done. This is essential whenever large numbers of samples are processed. Our software is also the only one to summarize all analysis results into user accounts with ready-to-deliver web reports. An overview of the features of several NGS workflow systems compared to QuickNGS is given in Table 3.

\section{Conclusions}

We have contributed QuickNGS, a software which extremely reduces the efforts put into basic data processing for Next-Generation Sequencing. QuickNGS takes advantage of powerful hardware architectures and a comprehensive database to control the overall workflow. As a result, our approach enables laboratories with a high throughput of NGS data analyses to now accomplish their basic bioinformatics work on next-generation sequencing data essentially in zero time.

\section{Availability and requirements}

Project home page: http://bifacility.uni-koeln.de/quickngs/ web

Operating system: Linux

Programming languages: Bash scripting, Perl, $\mathrm{R}$

Other requirements: MySQL server, Apache web server on separate machine

License: GNU GPL version 3

\section{Additional files}

Additional file 1: List of genes differentially expressed between the atf3a mutants and the yw controls in our test dataset. The foldchanges and p-values are produced from the DESeq2 package, whereas the FPKM values are taken from Cufflinks output. The file contains multiple tabs representing lists cut at particular thresholds for fold-change and p-value.

Additional file 2: List of exons differentially used between the atf3a mutants and the yw controls according to the DEXSeq package. The file contains multiple tabs representing lists cut at particular thresholds for fold-change and $p$-value.

Additional file 3: List containing the same $p$-values and foldchanges as Additional file 1 plus $p$-values and fold-changes for all other comparisons performed in the current QuickNGS runs. In this example, these are the comparisons of the three other mutant condition against wild type. This table facilitates comparisons between the genes differentially regulated under one condition and other conditions.

Additional file 4: Procedure to upload sample meta data into the QuickNGS database. (a) First, the file locations of the raw data need to be saved into a text file. (b) Together with information on library type, NGS application, species and laboratory, this file can be uploaded into the
QuickNGS web interface. (c) Human-readable sample labels as well as batch information can be provided for each sample listed in the text file. (d) Pairs of samples or sample groups can be defined for comparative analysis within the workflow.

\section{Abbreviations}

NGS: Next-Generation Sequencing; DB: Database; QC: Quality check; IT: Information technology; SQL: Structured query language; ChIP: Chromatin immunoprecipitation.

\section{Competing interests}

The authors declare that they have no competing interests.

\section{Authors' contributions}

PW, MN and PF developed the code. PW tested and debugged the code in high-throughput production mode. PF and MN wrote the manuscript. All authors read and approved the final manuscript.

\section{Acknowledgements}

This work was supported by the German Ministry of Economy and Energy (BMWi, Transcriptalyzer KF2429610MS2), the German Research Foundation (DFG, CancerSysDB FR3313/2-1), and the German Ministry for Education and Research (BMBF, NGSgoesHPC 011H11003A).

\section{Author details}

${ }^{1}$ Bioinformatics Core Facility, CECAD Research Center, University of Cologne, Joseph-Stelzmann-Str. 26, 50931 Cologne, Germany. ${ }^{2}$ Center for Molecular Medicine, University of Cologne, Robert-Koch-Str. 21, Cologne 50931, Germany.

Received: 5 October 2014 Accepted: 12 June 2015

Published online: 01 July 2015

\section{References}

1. Anders S, Huber W. Differential expression analysis for sequence count data. Genome Biol. 2010;11(10):R106.

2. Anders $\mathrm{S}$, Reyes $\mathrm{A}$, Huber W. Detecting differential usage of exons from RNA-seq data. Genome Res. 2012;22(10):2008-17.

3. Cingolani P, Patel VM, Coon M, Nguyen T, Land SJ, Ruden DM, Lu X. Using Drosophila melanogaster as a Model for Genotoxic Chemical Mutational Studies with a New Program, SnpSift. Front Genet. 2012;3:35.

4. Durinck S, Moreau Y, Kasprzyk A, Davis S, De Moor B, Brazma A, Huber W. BioMart and Bioconductor: a powerful link between biological databases and microarray data analysis. Bioinformatics. 2005;21(16):3439-40.

5. Feng J, Liu T, Qin B, Zhang Y, Liu XS. Identifying ChIP-seg enrichment using MACS. Nat Protoc. 2012;7(9):1728-40.

6. Friedländer MR, Chen W, Adamidi C, Maaskola J, Einspanier R, Knespel S, Rajewsky N. Discovering microRNAs from deep sequencing data using miRDeep. Nat Biotechnol. 2008;26(4):407-15.

7. Giardine B, Riemer C, Hardison RC, Burhans R, Elnitski L, Shah P, Zhang Y, Blankenberg D, Albert I, Taylor J, Miller W, Kent WJ, Nekrutenko A. Galaxy: a platform for interactive large-scale genome analysis. Genome Res. 2005;15(10):1451-5.

8. Griffiths-Jones S, Grocock RJ, van Dongen S, Bateman A, Enright AJ. miRBase: microRNA sequences, targets and gene nomenclature. Nucleic Acids Res. 2006;34(Database issue):D140-4.

9. Hubbard T, Barker D, Birney E, Cameron G, Chen Y, Clark L, Cox T, Cuff J, Curwen V, Down T, Durbin R, Eyras E, Gilbert J, Hammond M, Huminiecki L, Kasprzyk A, Lehvaslaiho H, Lijnzaad P, Melsopp C, Mongin E, Pettett R, Pocock M, Potter S, Rust A, Schmidt E, Searle S, Slater G, Smith J, Spooner W, Stabenau A, Stalker J, Stupka E, Ureta-Vidal A, Vastrik I, Clamp M. The Ensembl genome database project. Nucleic Acids Res. 2002;30(1):38-41.

10. Kallio MA, Tuimala JT, Hupponen T, Klemelä P, Gentile M, Scheinin I, Koski M, Käki J, Korpelainen El. Chipster: user-friendly analysis software for microarray and other high-throughput data. BMC Genomics. 2011;12:507.

11. Kent WJ, Sugnet CW, Furey TS, Roskin KM, Pringle TH, Zahler AM, Haussler D. The human genome browser at UCSC. Genome Res. 2002;12(6):996-1006. 
12. Kim D, Pertea G, Trapnell C, Pimentel H, Kelley R, Salzberg SL. TopHat2: accurate alignment of transcriptomes in the presence of insertions, deletions and gene fusions. Genome Biol. 2013;14(4):R36.

13. Li H, Durbin R. Fast and accurate short read alignment with BurrowsWheeler transform. Bioinformatics. 2009;25(14):1754-60.

14. Li H, Handsaker B, Wysoker A, Fennell T, Ruan J, Homer N, Marth G, Abecasis G, Durbin R. 1000 Genome Project Data Processing Subgroup (2009): The Sequence Alignment/Map format and SAMtools. Bioinformatics. 2009;25(16):2078-9.

15. Machanick P, Bailey TL. MEME-ChIP: motif analysis of large DNA datasets. Bioinformatics. 2011;27(12):1696-7.

16. Rausch T, Zichner T, Schlattl A, Stütz AM, Benes V, Korbel JO. DELLY: structural variant discovery by integrated paired-end and split-read analysis. Bioinformatics. 2012;28(18):i333-9.

17. Reich M, Liefeld T, Gould J, Lerner J, Tamayo P, Mesirov JP. GenePattern 2.0. Nat Genet. 2006;38(5):500-1.

18. Rynes J, Donohoe CD, Frommolt P, Brodesser S, Jindra M, Uhlirova M. Activating transcription factor 3 regulates immune and metabolic homeostasis. Mol Cell Biol. 2012;32(19):3949-62.

19. Trapnell C, Williams BA, Pertea G, Mortazavi A, Kwan G, van Baren MJ, Salzberg SL, Wold BJ, Pachter L. Transcript assembly and quantification by RNA-Seq reveals unannotated transcripts and isoform switching during cell differentiation. Nat Biotechnol. 2010;28(5): 511-5

\section{Submit your next manuscript to BioMed Central and take full advantage of:}

- Convenient online submission

- Thorough peer review

- No space constraints or color figure charges

- Immediate publication on acceptance

- Inclusion in PubMed, CAS, Scopus and Google Scholar

- Research which is freely available for redistribution 